\title{
Article \\ Performance Analysis of AF Cooperative Relaying Networks with SWIPT
}

\author{
Zih-Sin Wang ${ }^{1}$, Liang-Hung Lin ${ }^{1}$, Jyh-Horng Wen ${ }^{2}$, Yen-Ju Lin ${ }^{3}$ and Chien-Erh Weng ${ }^{3, *}$ (i) \\ 1 School of Software and Microelctronics, Peking University, Beijing 100871, China; \\ hank6112003_2@yahoo.com (Z.-S.W.); ceweng0126@gmail.com (L.-H.L.) \\ 2 Department of Electrical Engineering, Tunghai University, Taichung 407224, Taiwan; jhwen@thu.edu.tw \\ 3 Department of Telecommunication Engineering, National Kaohsiung University of Science and Technology, \\ Kaohsiung 81157, Taiwan; f109185106@nkust.edu.tw \\ * Correspondence: ceweng@nkust.edu.tw
}

Citation: Wang, Z.-S.; Lin, L.-H.; Wen, J.-H.; Lin, Y.-J.; Weng, C.-E. Performance Analysis of AF Cooperative Relaying Networks with SWIPT. Electronics 2022, 11, 589. https://doi.org/10.3390/ electronics11040589

Academic Editor: Raed

A. Abd-Alhameed

Received: 30 December 2021 Accepted: 13 February 2022 Published: 15 February 2022

Publisher's Note: MDPI stays neutral with regard to jurisdictional claims in published maps and institutional affiliations.

Copyright: (c) 2022 by the authors. Licensee MDPI, Basel, Switzerland. This article is an open access article distributed under the terms and conditions of the Creative Commons Attribution (CC BY) license (https:/ / creativecommons.org/licenses/by/ $4.0 /)$.

\begin{abstract}
Cooperative communication networks have received more attention due to its ability to improve the signal quality of terminal devices by spatial diversity. Under recent advance in internet of things, In order to extend the service life of terminal devices powered by battery, simultaneous wireless information and power transfer (SWIPT) technique has been emphasize. The terminal devices can harvest energy and decode information from the same radio frequency (RF) signal using by SWIPT technique. In this paper, we combine both techniques to study the performance of both conventional cooperative relaying networks without SWIPT and cooperative relaying networks with SWIPT under an amplify-and-forward (AF) relaying network. To the best of our knowledge, no one simultaneously studies and compares the performance of both systems. Therefore, the outage probabilities of both systems are carried out, and numerical results are compared in this paper. The main results include: (1) Compared with conventional cooperative communication, the cooperative communication with SWIPT has better outage probability only when the distance between relay node and source node is less than one. It implies that, to outperform the conventional cooperative communication, the relay node should harvest enough energy for signal transmission. (2) With the diversity of direct path and relay path, the outage probability of cooperative communication with $\mathrm{EH}$ under an AF relaying network has been significantly reduced.
\end{abstract}

Keywords: cooperative communication; SWIPT; amplify-and-forward; outage probability

\section{Introduction}

In recent years, more and more research on multi-antenna systems has been study, since multi-antenna technology can provide spatial diversity, which greatly improves the quality, speed, and range of communication systems. However, considering to device size, cost and hardware limitations, some scenarios like the node device of Internet of Thing may not be able to support multiple transmit antennas. As a result, cooperative communication has been emphasized. Cooperative communication can send signals to the destination node through the antennas of other devices as relay nodes to achieve the effect of virtual multi-antennas [1]. In the application environment of the Internet of Things that, the terrain is steep, and the number is so large that it is very infeasible to change the battery. Hence, energy harvesting $(\mathrm{EH})$ to extend the service life of the node has become an important issue [2]. Traditional EH methods are based on natural sources such as solar, wind, vibrations, ambient radio frequency (RF) signals, etc. Among the above energy sources, only RF signals can generate energy relatively stably (by RF signals emitted from the surroundings), thus attracting many research interests of scholars [3,4]. Moreover, simultaneous wireless information and power transfer (SWIPT), which is the most prominent technique, as it provides spectral efficiency by delivering energy and information to the relays at the same time was further proposed by academic [2,5-8]. 
The integration of cooperative relay and simultaneous wireless information and power transfer (SWIPT) techniques has evolved as a new phenomenon for the next-generation wireless communication system in [9]. Cooperative relay technique is used to get spectral efficient network and energy and to solve the issues of fading, shadowing, path loss, and smaller coverage area. In [10] investigated the performance of cache-assisted simultaneous wireless information and power transfer (SWIPT) cooperative systems, in which one source communicates with one destination via the aid of multiple relays. The energy efficiency performance of a simultaneous wireless information and power transfer (SWIPT)-enabled cooperative relay network in the presence of an interfering transmitter (IT) was investigated, and the optimal transmitted source's power for each scenario and obtained the suboptimal power splitting and energy consumed factors was derived in [11]. Three more feasible SWIPT receiver architectures, such as time switching (TS), power splitting (PS) and antenna switching (AS) were proposed in [12]. Considering that under TS, the short time slot that is cut out makes it difficult for the amplifier to maintain linearity and refers to the case of considering a single antenna, we adopt PS receiver architectures of SWIPT in this paper.

In [6], an amplify-and-forward (AF) wireless cooperative relaying network has been considered. Authors propose the TSR and the PSR protocols based on the TS and PS receiver architectures for energy-constrained relay in wireless AF relaying networks. The throughput performance under both TS and PS receiver architectures in SWIPT are carried out. However, there is no direct path in [6], hence, reference [7] investigated the outage probability performance of an AF cooperative relaying network under PS receiver architectures in SWIPT with direct path. The above articles only compare scenarios of cooperative relaying network with SWIPT under different conditions.

In this paper, we consider an AF cooperative relaying network with one source node, one destination node, and one relay node. Both of conventional cooperative relaying networks without SWIPT and cooperative relaying networks with SWIPT are considered. We also consider the case of assistance with the direct path and the case of no direct path in the above scenarios. In the scenario where direct path assistance is considered, the destination uses maximum ratio combining (MRC) to combine the signals from source node to destination node and the signals from relay node to destination node. The power splitting-based relaying (PSR) protocol in [6] which based on the PS receiver architecture, is considered for the relay node under scenario of SWIPT. Numerical results show that cooperative relay networks with SWIPT are superior to cooperative relay networks without SWIPT under harvested sufficient energy.

The rest of the paper is organized as follows: the system model is described in Section 2. Sections 3 and 4 detail the outage behavior of the system model under conventional cooperative relaying networks without SWIPT and cooperative relaying networks with SWIPT, respectively. Section 5 presents the numerical results. Finally, Section 6 concludes the paper.

\section{System Model}

We consider a dual-hop AF relaying cooperative network, where the signal from a source node (S) to a destination node (D) through the relay node (R). As illustrated in Figure $1 \mathrm{a}, \mathrm{b}$, the system without and with direct path are considered, respectively. All nodes are equipped with a single antenna and operated in a half-duplex mode. 
(a)

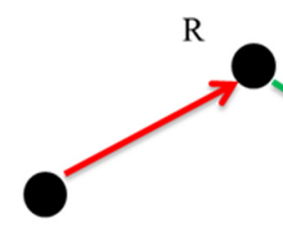

$\mathrm{S}$

(b)

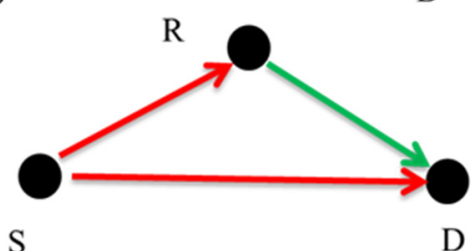

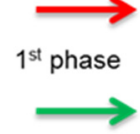

$2^{\text {nd }}$ phase

Figure 1. The system model without SWIPT. (a) the signal from a source to a destination through the relay; (b) the signal from a source to a destination through the relay with direct path.

In the first phase, the signal is transmitted from $S$ to both $R$ and $D$. The received signal at $R$ and $D$ can be expressed as: In the first phase, the signal is transmitted from $S$ to both $R$ and $D$. The received signal at $R$ and $D$ can be expressed as:

$$
\begin{aligned}
& y_{s r}=\sqrt{\frac{P_{s}}{d_{s r}^{k}}} h_{s r} x+n_{s r} \\
& y_{s d}=\sqrt{\frac{P_{s}}{d_{s d}^{k}}} h_{s d} x+n_{s d}
\end{aligned}
$$

where $P_{S}$ is the transmission powers of $\mathrm{S}, d_{s r}$ and $d_{s d}$ are the distances from $\mathrm{S}$ to $\mathrm{R}$ and $\mathrm{S}$ to $\mathrm{D}$, respectively, $h_{s r}$ and $h_{s d}$ are the channel gains of the aforesaid two paths, which are independent complex Gaussian random variable with mean is zero, and its variances are $\sigma_{s r}^{2}$ and $\sigma_{s d}^{2}$, respectively, $k$ is the path loss exponent, $x$ is the normalized information signal from $\mathrm{S}$, i.e., $\mathrm{E}\left[|x|^{2}\right]=1$, where $\mathrm{E}[\cdot]$ stands for the expectation operation, $|\cdot|$ stands for the absolute value operation, $n_{s r}$ and $n_{s d}$ are the zero mean additive white Gaussian noise (AWGN) with variances are $N_{s r}$ and $N_{s d}$, respectively.

In the second phase, the transmitted signal from $\mathrm{R}$ to $\mathrm{D}, x_{r}$, is amplified and normalized from the signal $y_{s r}$ by AF relaying strategy, i.e., $x_{r}=\alpha y_{s r}, \mathrm{E}\left[\left|x_{r}\right|^{2}\right]=1$, where the amplifying factor $\alpha$ is defined as:

$$
\alpha=\sqrt{\frac{1}{\left(\frac{P_{s}}{d_{s r}^{k}}\left|h_{s r}\right|^{2}+N_{s r}\right)}}
$$

Then the received signal at $\mathrm{D}$ in the second phase can be expressed as:

$$
y_{r d}=\sqrt{\frac{P_{r}}{d_{r d}^{k}}} h_{r d} x_{r}+n_{r d}
$$

where $P_{r}$ is the transmission powers of $\mathrm{R}, d_{r d}$ is the distances from $\mathrm{R}$ to $\mathrm{D}, h_{r d}$ is channel gain of $\mathrm{R}$ to $\mathrm{D}$ which is complex Gaussian random variable with a mean is zero, and variance is $\sigma_{r d}^{2}, n_{r d}$ is the zero mean AWGN with variance is $N_{r d}$. Substituting $x_{r}=\alpha y_{s r}$ and (3) into (4), the received signal at $\mathrm{D}$ can be further rewritten as:

$$
y_{r d}=\sqrt{\frac{P_{r} P_{s}}{\left(\frac{P_{s}}{d_{s r}^{k}}\left|h_{s r}\right|^{2}+N_{s r}\right) d_{s r}^{k} d_{r d}^{k}}} h_{s r} h_{r d} x+n_{r d}+\sqrt{\frac{P_{r}}{\left(\frac{P_{s}}{d_{s r}^{k}}\left|h_{s r}\right|^{2}+N_{s r}\right) d_{r d}^{k}}} h_{r d} n_{s r}
$$


According to (5), the signal to noise ratio (SNR) of conventional cooperative communication without direct path can be expressed as:

$$
\begin{aligned}
& \gamma_{A F, n o d i r}=\frac{\frac{P_{r} P_{S}}{\left(\frac{P_{S}}{d_{s r}^{k}}\left|h_{s r}\right|^{2}+N_{s r}\right) d_{s r}^{k} d_{r d}^{k}}\left|h_{s r}\right|^{2}\left|h_{r d}\right|^{2}}{\left(\frac{P_{S}}{\left.\frac{P_{r}}{d_{s r}^{k}}\left|h_{S r}\right|^{2}+N_{s r}\right) d_{r d}^{k}}\left|h_{r d}\right|^{2} N_{s r}+N_{r d}\right.} \\
& =\frac{\frac{P_{r} P_{s}}{d_{s r}^{k} d_{r d}^{k}}\left|h_{s r}\right|^{2}\left|h_{r d}\right|^{2}}{\frac{P_{r}}{d_{r d}^{k}}\left|h_{r d}\right|^{2} N_{s r}+\frac{P_{s}}{d_{s r}^{k}}\left|h_{s r}\right|^{2} N_{r d}+N_{s r} N_{r d}} \\
& =\frac{\gamma_{s r} \gamma_{r d}}{\gamma_{s r}+\gamma_{r d}+1}
\end{aligned}
$$

where $\gamma_{s r}=\frac{P_{s}\left|h_{s r}\right|^{2}}{d_{s r}^{k} N_{s r}}, \gamma_{r d}=\frac{P_{r}\left|h_{r d}\right|^{2}}{d_{r d}^{k} N_{r d}}$.

In the case, which consider with direct path, the SNR can be increased through combining the received signals $y_{s d}$ and $y_{r d}$. The maximum ratio combining (MRC) of combining technique is applied in this paper. As a result, the signal to noise ratio (SNR) of conventional cooperative communication with direct path can be expressed as:

$$
\gamma_{A F, \text { dir }}=\gamma_{A F, \text { no dir }}+\gamma_{s d}
$$

where $\gamma_{s d}=\frac{P_{s}\left|h_{s d}\right|^{2}}{d_{s d}^{k} N_{s d}}$

From information theory [13], the achievable capacity of direct path can be expressed as:

$$
C_{d i r}=\log _{2}\left(1+\gamma_{s d}\right)
$$

Therefore, the achievable capacity of conventional cooperative communication can be expressed as:

$$
\begin{aligned}
C_{A F, \text { no dir }} & =\frac{1}{2} \log _{2}\left(1+\gamma_{A F, \text { no dir }}\right), \\
C_{A F, \text { dir }} & =\frac{1}{2} \log _{2}\left(1+\gamma_{A F, \text { dir }}\right),
\end{aligned}
$$

where $C_{A F, \text { no dir }}$ and $C_{A F, \text { dir }}$ are the achievable capacity without direct path and the achievable capacity with direct path, respectively.

In the other hand, the system model with SWIPT as illustrated in Figure 2, where the signal from $S$ to $D$ through $E H$ relay node (EHR). We consider PSR protocol for the AF EHR. The radio frequency $(\mathrm{RF})$ received signal at EHR in the first phase can be represented mathematically as:

$$
y_{s r, R}=\sqrt{\frac{P_{s}}{d_{s r}^{k}}} h_{s r} x+n_{s r, R},
$$

where $n_{s r, R}$ is the zero mean AWGN from $S$ to RF band of EHR with variance is $N_{s r, R}$. According to PSR protocol, partially received signal at the EHR is used for energy harvesting $(\mathrm{EH})$. The harvested energy is obtained as:

$$
E_{h}=\eta \rho \frac{P_{s}}{d_{s r}^{k}}\left|h_{s r}\right|^{2} \frac{T}{2}
$$

where $0 \leq \eta \leq 1$ is the energy conversion efficiency, $0 \leq \rho \leq 1$ is the power splitting factor, $T$ is the total transmission time. Since EH by the noise is a small constant and therefore ignored. With collected energy $E_{h}$, the transmission powers of EHR can be expressed as:

$$
P_{r}=\frac{E_{h}}{T / 2}=\eta \rho \frac{P_{s}}{d_{s r}^{k}}\left|h_{s r}\right|^{2}
$$


Consider the RF signal received by EHR with PSR protocol and the noise due to RF band to baseband signal conversion, the received information signal at the EHR in the first phase is represented as:

$$
y_{s r, \mathrm{EH}}=\sqrt{\frac{P_{s}(1-\rho)}{d_{s r}^{k}}} h_{s r} x+\sqrt{(1-\rho)} n_{s r, R}+n_{s r, C}=\sqrt{\frac{P_{s}(1-\rho)}{d_{s r}^{k}}} h_{s r} x+\widetilde{n}_{s r},
$$

where $n_{s r, C}$ is the zero mean AWGN from RF band to baseband signal conversion at EHR with variance is $N_{s r, C}, \widetilde{n}_{s r}=\sqrt{(1-\rho)} n_{s r, R}+n_{s r, C}$, with variance is $\widetilde{N}_{s r}=(1-\rho) N_{s r, R}+N_{s r, C}$. For a fair comparison of conventional cooperative communication without energy harvesting and cooperative communication with energy harvesting, we assume that $N_{s r}=N_{s r, R}+N_{s r, C}$. In the second phase, the transmitted signal from EHR to D, $x_{r, \mathrm{EH}}$, is amplified and normalized from the signal $y_{s r}, \mathrm{EH}$ by AF relaying strategy, i.e., $x_{r, \mathrm{EH}}=\alpha_{\mathrm{EH}} y_{s r, \mathrm{EH}}$, $\mathrm{E}\left[\left|x_{r, \mathrm{EH}}\right|^{2}\right]=1$, where the amplifying factor $\alpha_{\mathrm{EH}}$ is defined as:

$$
\alpha_{\mathrm{EH}}=\sqrt{\frac{1}{\left(\frac{P_{s}}{d_{s r}^{k}}\left|h_{s r}\right|^{2}+\tilde{N}_{s r}\right)}}
$$

Then the received signal at $\mathrm{D}$ in the second phase can be expressed as:

$$
y_{r d, \mathrm{EH}}=\sqrt{\frac{P_{r}}{d_{r d}^{k}}} h_{r d} x_{r, \mathrm{EH}}+n_{r d}
$$

Substituting $x_{r, \mathrm{EH}}=\alpha_{\mathrm{EH}} y_{s r, \mathrm{EH}}$ and (14) into (16), the received signal at $\mathrm{D}$ can be further rewritten as:

$$
y_{r d}=\sqrt{\frac{P_{r} P_{s}}{\left(\frac{P_{s}}{d_{s r}^{k}}\left|h_{s r}\right|^{2}+N_{s r}\right) d_{s r}^{k} d_{r d}^{k}}} h_{s r} h_{r d} x+n_{r d}+\sqrt{\frac{P_{r}}{\left(\frac{P_{s}}{d_{s r}^{k}}\left|h_{s r}\right|^{2}+N_{s r}\right) d_{r d}^{k}}} h_{r d} n_{s r} .
$$

According to (17), the SNR of cooperative communication without a direct path under PSR protocol can be expressed as:

$$
\begin{aligned}
& \gamma_{A F, \mathrm{EH}, n o d i r}=\frac{\frac{\eta \rho P_{S}{ }^{2}\left|h_{s r}\right|^{2}(1-\rho)}{\left(\frac{P_{S}(1-\rho)}{d_{s r}^{k}}\left|h_{s r}\right|^{2}+\widetilde{N}_{s r}\right) d_{r d}^{k} d_{s r}^{2 k}}\left|h_{s r}\right|^{2}\left|h_{r d}\right|^{2}}{\frac{\eta \rho P_{s}\left|h_{s r}\right|^{2}}{\left(\frac{P_{S}(1-\rho)}{d_{s r}^{k}}\left|h_{s r}\right|^{2}+\widetilde{N}_{s r}\right) d_{r d}^{k} d_{s r}^{k}}\left|h_{r d}\right|^{2} \widetilde{N}_{s r}+N_{r d}} \\
& =\frac{\eta(1-\rho) \rho P_{s}^{2}\left|h_{s r}\right|^{4}\left|h_{r d}\right|^{2}}{\eta \rho P_{s}\left|h_{s r}\right|^{2}\left|h_{r d}\right|^{2} d_{s r}^{k} \widetilde{N}_{s r}+(1-\rho) P_{s}\left|h_{s r}\right|^{2} d_{r d}^{k} d_{s r}^{k} N_{r d}+d_{s r}^{2 k} d_{r d}^{k} \widetilde{N}_{s r} N_{r d}} \\
& =\frac{\gamma_{s r, \mathrm{EH}} \gamma_{r d, \mathrm{EH}}}{\gamma_{s r, \mathrm{EH}}+\gamma_{r d, \mathrm{EH}}+1}
\end{aligned}
$$

where $\gamma_{s r, \mathrm{EH}}=\frac{(1-\rho) P_{s}\left|h_{s r}\right|^{2}}{d_{s r}^{k} \widetilde{N}_{s r}}, \gamma_{r d, \mathrm{EH}}=\frac{P_{r}\left|h_{r d}\right|^{2}}{d_{r d}^{k} N_{r d}}=\frac{\eta \rho P_{s}\left|h_{s r}\right|^{2}\left|h_{r d}\right|^{2}}{d_{s r}^{k} d_{r d}^{k} N_{r d}}$. Moreover, the SNR of cooperative communication with direct path under PSR protocol can be expressed as:

$$
\gamma_{A F, \mathrm{EH}, \mathrm{dir}}=\gamma_{A F, \mathrm{EH}, \text { no dir }}+\gamma_{s d}
$$

The achievable capacity of cooperative communication with EH can be expressed as:

$$
\begin{gathered}
C_{A F, \mathrm{EH}, \text { no dir }}=\frac{1}{2} \log _{2}\left(1+\gamma_{A F, \mathrm{EH}, \text { no dir }}\right), \\
C_{A F, \mathrm{EH}, \text { dir }}=\frac{1}{2} \log _{2}\left(1+\gamma_{A F, \mathrm{EH}, \text { dir }}\right),
\end{gathered}
$$


where $C_{A F, E H, n o \text { dir }}$ and $C_{A F, E H, d i r}$ are the achievable capacity under PSR protocol without direct path and the achievable capacity with direct path.

(a)

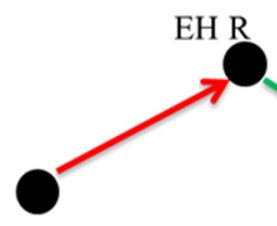

S

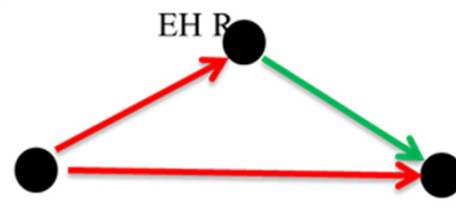

$\mathrm{S}$

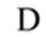

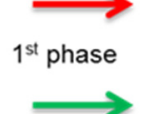

$2^{\text {nd }}$ phase

(b)

Figure 2. The system model with EH. (a) the signal from a source to a destination through the relay; (b) the signal from a source to a destination through the relay with direct path.

\section{Analysis of Optimal Outage Probability under Relay without EH}

In this section, we evaluate the optimal outage probability of conventional cooperative communication with direct path or not. In the case, which consider without direct path, the optimization problem can be presented mathematically as:

$$
\begin{aligned}
& \min _{0 \leq d_{s r} \leq d_{s d}} P_{\text {out }}=\min _{0 \leq d_{s r} \leq d_{s d}} \operatorname{Pr}\left[C_{A F, \text { no dir }} \leq R\right], \\
& 0 \leq P_{s} \leq P_{t} \quad 0 \leq P_{s} \leq P_{t}
\end{aligned}
$$

Subject to

$$
\begin{aligned}
d_{s r}+d_{r d} & =d_{s d}, \\
P_{s}+P_{r} & =P_{t},
\end{aligned}
$$

where $P_{t}$ is the total transmit power, $R$ is the data rate threshold for the outage probability.

First, the cumulative distribution function (CDF) of $C_{A F, n o \text { dir }}$ is given by:

$$
F_{C_{A F, n o d i r}}(R)=\operatorname{Pr}\left[C_{A F, \text { no dir }} \leq R\right]=\operatorname{Pr}\left[\gamma_{A F, \text { no dir }} \leq 2^{2 R}-1\right] .
$$

At high SNR, (25) can be simplified as [10]:

$$
\begin{gathered}
F_{C_{A F, \text { no dir }}}(R) \approx \operatorname{Pr}\left[\frac{\gamma_{s r} \gamma_{r d}}{\gamma_{s r}+\gamma_{r d}} \leq 2^{2 R}-1\right] \\
=1-\exp \left\{-\left(\frac{2^{2 R}-1}{\gamma_{s r, \text { mean }}}+\frac{2^{2 R}-1}{\gamma_{r d, \text { mean }}}\right)\right\} \frac{2\left(2^{2 R}-1\right)}{\sqrt{\gamma_{s r} \text {,mean }} \gamma_{r d, \text { mean }}} \mathrm{K}_{1}\left(\frac{2\left(2^{2 R}-1\right)}{\sqrt{\gamma_{s r}, \text { mean }} \gamma_{r d, \text { mean }}}\right),
\end{gathered}
$$

where $\gamma_{s r, \text { mean }}=\frac{P_{s}}{\lambda_{s r} d_{s r}^{k} N_{s r}}$ is the expected value of $\gamma_{s r}, \gamma_{s r, \text { mean }}=\frac{P_{s}}{\lambda_{s r} d_{s r}^{k} N_{s r}}$ is the expected value of $\gamma_{s r}, \lambda_{s r}=\frac{1}{\sigma_{s r}^{2}}, \lambda_{r d}=\frac{1}{\sigma_{r d}^{2}}, \mathrm{~K}_{1}(\cdot)$ is the first order modified Bessel function of the second kind. At high SNR, $\frac{2\left(2^{2 R}-1\right)}{\sqrt{\gamma_{s r} \text {,mean } \gamma_{r d, \text { mean }}}} \ll 1$, so $\mathrm{K}_{1}\left(\frac{2\left(2^{2 R}-1\right)}{\sqrt{\gamma_{s r} \text {,mean }} \gamma_{r d, \text { mean }}}\right)$ can be approximated as $\frac{\sqrt{\gamma_{\text {sr }, \text { mean }} \gamma_{\text {rd, mean }}}}{2\left(2^{2 R}-1\right)}[11]^{9.6 .9}$. As a result, (26) can be further rewritten as:

$$
\begin{aligned}
& F_{C_{A F, n \text { dir }}}(R) \approx 1-\exp \left\{-\left(\frac{2^{2 R}-1}{\gamma_{s r}, \text { mean }}+\frac{2^{2 R}-1}{\gamma_{r d, \text { mean }}}\right)\right\} \\
& =1-\exp \left\{-\left(\frac{N_{s s} d_{s s}^{k} \lambda_{s r}}{P_{s}}+\frac{N_{r d} d_{r d}^{k} \lambda_{r d}}{P_{r}}\right)\left(2^{2 R}-1\right)\right\} .
\end{aligned}
$$


We can observe from (27) that the outage probability is monotone decreasing with $\left(\frac{N_{s r} d_{s r}^{k} \lambda_{s r}}{P_{s}}+\frac{N_{r d} d_{r d}^{k} \lambda_{r d}}{P_{r}}\right)$. Thus, the optimal parameters of (22), $\hat{d}_{s r}$ and $\hat{P}_{s}$, can be obtained as:

$$
\begin{gathered}
\left(\hat{d}_{s r}, \hat{P}_{s}\right)=\arg \min _{\substack{0 \leq d_{s r} \leq d_{s d} \\
0 \leq P_{s} \leq P_{t}}}\left(\frac{N_{s r} d_{s r}^{k} \lambda_{s r}}{P_{s}}+\frac{N_{r d} d_{r d}^{k} \lambda_{r d}}{P_{r}}\right) \\
=\arg \min _{0 \leq d_{s r} \leq d_{s d}}\left(\frac{N_{s r} d_{s r}^{k} \lambda_{s r}}{P_{s}}+\frac{N_{r d}\left(d_{s d}-d_{s r}\right)^{k} \lambda_{r d}}{P_{t}-P_{s}}\right) \\
0 \leq P_{s} \leq P_{t}
\end{gathered}
$$

Let $f_{1}\left(d_{s r}, P_{s}\right) \equiv \frac{N_{s r} d_{s r}^{k} \lambda_{s r}}{P_{s}}+\frac{N_{r d}\left(d_{s d}-d_{s r}\right)^{k} \lambda_{r d}}{P_{t}-P_{s}}$. By taking $\frac{\partial f_{1}}{\partial d_{s r}}=0$ and $\frac{\partial f_{1}}{\partial P_{s}}=0$, and after some manipulation, we obtain:

$$
\begin{gathered}
\hat{d}_{s r}=\frac{d_{s d}}{1+\left(\frac{N_{s r} \lambda_{s r}}{N_{r d} \lambda_{r d}}\right)^{\frac{1}{k-2}}}, \\
\hat{P}_{S}=\frac{P_{t}}{1+\left(\frac{N_{s r} \lambda_{s r}}{N_{r d} \lambda_{r d}}\right)^{\frac{1}{k-2}}} .
\end{gathered}
$$

Replace (29) and (30) into (22), the optimal outage probability of conventional cooperative communication without direct path under high SNR is obtained as:

$$
\min _{\substack{0 \leq d_{s r} \leq d_{s d} \\ 0 \leq P_{s} \leq P_{t}}} P_{\text {out }} \approx 1-\exp \left\{-\left(\frac{N_{s r} d_{s d}{ }^{k} \lambda_{s r}\left(2^{2 R}-1\right)}{\left(1+\left(\frac{N_{s r} \lambda_{s r}}{N_{r d} \lambda_{r d}}\right)^{\frac{1}{k-2}}\right)^{k-2} P_{t}}\right)\right\} .
$$

In the case, which consider with direct path, the optimization problem can be presented mathematically as:

$$
\begin{gathered}
\min _{0 \leq d_{s r} \leq d_{s d}} \quad P_{\text {out }}=\quad \min _{0 \leq d_{s r} \leq d_{s d}} \operatorname{Pr}\left[C_{A F, \text { dir }} \leq R\right] \\
0 \leq P_{s} \leq P_{t}
\end{gathered}
$$

Subject to

$$
\begin{gathered}
d_{s r}+d_{r d}=d_{s d}, \\
P_{s}+P_{r}=P_{t} .
\end{gathered}
$$

First, the cumulative distribution function $(\mathrm{CDF})$ of $C_{A F \text {, dir }}$ is given by:

$$
\begin{gathered}
F_{C_{A F, \text { dir }}}(x)=\operatorname{Pr}\left[C_{A F, \text { dir }} \leq R\right]=\operatorname{Pr}\left[\gamma_{s d}+\gamma_{A F, \text { no dir }} \leq 2^{2 R}-1\right] \\
\approx 1+\frac{\frac{\gamma_{s d, \text { mean }}}{\gamma_{s r \text {,mean }}}+\frac{\gamma_{s d, \text { mean }}}{\gamma_{r d, \text { mean }}}}{1-\frac{\gamma_{s d, \text { mean }}}{\gamma_{s r, \text { mean }}}-\frac{\gamma_{\text {sd, mean }}}{\gamma_{r d, \text { mean }}}} \exp \left\{-\frac{2^{2 R}-1}{\gamma_{s d, \text { mean }}}\right\}-\frac{\exp \left\{-\left(\frac{2^{2 R}-1}{\gamma_{s r, \text { mean }}}+\frac{2^{2 R}-1}{\gamma_{r d, \text { mean }}}\right)\right\}}{1-\frac{\gamma_{s d, \text { mean }}}{\gamma_{s r} \text {,mean }}-\frac{\gamma_{s d, \text { mean }}}{\gamma_{r d, \text { mean }}}},
\end{gathered}
$$

where $\gamma_{s d, \text { mean }}=\frac{P_{s}}{\lambda_{s d} d_{s d}^{k} N_{s d}}$ is the expected value of $\gamma_{s d}$. At high SNR, $\frac{2^{2 R}-1}{\gamma_{s d, m e a n}} \ll 1$ and $\frac{2^{2 R}-1}{\gamma_{s r, \text { mean }}}+\frac{2^{2 R}-1}{\gamma_{r d \text {,mean }}} \ll 1$. As a result, (35) can be approximated by second order Taylor series expansion as:

$$
\begin{aligned}
& F_{C_{A F, \text { dir }}}(R) \approx \frac{1}{\gamma_{s d, \text { mean }}} \frac{\gamma_{s r, \text { mean }}+\gamma_{r d, \text { mean }}}{\gamma_{s r, \text { mean }} \gamma_{r d, \text { mean }}} \frac{\left(2^{2 R}-1\right)^{2}}{2} \\
& =\frac{\lambda_{s d} d_{s d}^{k} N_{s d}}{P_{s}}\left(\frac{\lambda_{s r} d_{s r}^{k} N_{s r}}{P_{s}}+\frac{\lambda_{r d} d_{r d}^{k} N_{r d}}{P_{r}}\right) \frac{\left(2^{2 R}-1\right)^{2}}{2} .
\end{aligned}
$$


We can observe from (27) that the outage probability is monotone decreasing with $\frac{1}{P_{s}}\left(\frac{\lambda_{s r} d_{s r}^{k} N_{s r}}{P_{s}}+\frac{\lambda_{r d} d_{r d}^{k} N_{r d}}{P_{r}}\right)$. Thus, the optimal parameters of (32), $\hat{d}_{s r}$ and $\hat{P}_{s}$, can be obtained as:

$$
\begin{gathered}
\left(\hat{d}_{s r}, \hat{P}_{s}\right)=\arg \min _{\substack{0 \leq d_{s r} \leq d_{s d} \\
0 \leq P_{s} \leq P_{t}}} \frac{1}{P_{s}}\left(\frac{\lambda_{s r} d_{s r}^{k} N_{s r}}{P_{s}}+\frac{\lambda_{r d} d_{r d}^{k} N_{r d}}{P_{r}}\right) \\
=\arg \min _{0 \leq d_{s r} \leq d_{s d}} \frac{1}{P_{s}}\left(\frac{\lambda_{s r} d_{s r}^{k} N_{s r}}{P_{s}}+\frac{\lambda_{r d}\left(d_{s d}-d_{s r}\right)^{k} N_{r d}}{\left(P_{t}-P_{s}\right)}\right) . \\
0 \leq P_{s} \leq P_{t}
\end{gathered}
$$

Let $f_{2}\left(d_{s r}, P_{s}\right) \equiv \frac{1}{P_{s}}\left(\frac{\lambda_{s r} d_{s r}^{k} N_{s r}}{P_{s}}+\frac{\lambda_{r d}\left(d_{s d}-d_{s r}\right)^{k} N_{r d}}{\left(P_{t}-P_{s}\right)}\right)$. By taking $\frac{\partial f_{2}}{\partial d_{s r}}=0$ and $\frac{\partial f_{2}}{\partial P_{s}}=0$, and after some manipulation, we obtain:

$$
\begin{gathered}
P_{s}=\frac{\lambda_{s r} d_{s r}^{k-1} N_{s r}}{\lambda_{r d}\left(d_{s d}-d_{s r}\right)^{k-1} N_{r d}+\lambda_{s r} d_{s r}^{k-1} N_{s r}} P_{t}, \\
\left(d_{s d}+d_{s r}\right) \lambda_{r d}\left(d_{s d}-d_{s r}\right)^{k-2} N_{r d}=\lambda_{s r} d_{s r}^{k-1} N_{s r} .
\end{gathered}
$$

Unfortunately, it is hard to find the closed-form expressions of this optimization problem. Therefore, the optimization problem result of outage probability can be found by numerical analysis the optimal parameters $\hat{d}_{s r}$ and $\hat{P}_{s}$. Replace $\hat{d}_{s r}$ and $\hat{P}_{s}$ into (31), the optimal outage probability of conventional cooperative communication with direct path under high SNR is obtained as:

$$
\min _{\substack{0 \leq d_{s r} \leq d_{s d} \\ 0 \leq P_{s} \leq P_{t}}} P_{\text {out }} \approx \frac{\lambda_{s d} d_{s d}^{k} N_{s d}}{\hat{P}_{s}}\left(\frac{\lambda_{s r} \hat{d}_{s r}^{k} N_{s r}}{\hat{P}_{s}}+\frac{\lambda_{r d}\left(d_{s d}-\hat{d}_{s r}\right)^{k} N_{r d}}{P_{t}-\hat{P}_{s}}\right) \frac{\left(2^{2 R}-1\right)^{2}}{2} .
$$

\section{Analysis of Optimal Outage Probability under Relay with EH}

In this section, we evaluate the optimal outage probability of EH cooperative communication with and without direct path. In the case which consider without direct path, the optimization problem can be presented mathematically as:

$$
\begin{gathered}
\min _{0 \leq d_{s r} \leq d_{s d}} \quad P_{\text {out }}= \\
0 \leq \rho \leq 1 \\
0 \leq d_{s r} \leq d_{s d} \\
0 \leq \rho \leq 1
\end{gathered}
$$

Subject to

$$
d_{s r}+d_{r d}=d_{s d}
$$

First, the CDF of $C_{A F, E H, n o \text { dir }}$ is given by

$$
\begin{gathered}
F_{C_{A F, \mathrm{EH}, n o d i r}}(R)=\operatorname{Pr}\left[C_{A F, \mathrm{EH}, \text { no dir }} \leq R\right]=\operatorname{Pr}\left[\gamma_{A F, \mathrm{EH}, \text { no dir }} \leq 2^{2 R}-1\right] \\
=\operatorname{Pr}\left[\frac{\eta(1-\rho) \rho P_{s}^{2}\left|h_{s r}\right|^{4}\left|h_{r r}\right|^{2}}{\eta \rho P_{s}\left|h_{s r}\right|^{2}\left|h_{r d}\right|^{2} d_{s r}^{k} \widetilde{N}_{s r}+(1-\rho) P_{s}\left|h_{s r}\right|^{2} d_{r d}^{k} d_{s r}^{k} N_{r d}+d_{s r}^{2 k} d_{r d}^{k} \widetilde{N}_{s r} N_{r d}} \leq 2^{2 R}-1\right] \\
=\operatorname{Pr}\left[\eta(1-\rho) \rho P_{s}{ }^{2}\left|h_{s r}\right|^{4}\left|h_{r d}\right|^{2}\right. \\
\leq \eta \rho P_{S}\left|h_{s r}\right|^{2}\left|h_{r d}\right|^{2} d_{s r}^{k} \widetilde{N}_{s r}\left(2^{2 R}-1\right) \\
\left.+(1-\rho) P_{s}\left|h_{s r}\right|^{2} d_{r d}^{k} d_{s r}^{k} N_{r d}\left(2^{2 R}-1\right)+d_{s r}^{2 k} d_{r d}^{k} \widetilde{N}_{s r} N_{r d}\left(2^{2 R}-1\right)\right] \\
=\operatorname{Pr}\left[a\left|h_{s r}\right|^{4}\left|h_{r d}\right|^{2} \leq b\left|h_{s r}\right|^{2}\left|h_{r d}\right|^{2}+c\left|h_{s r}\right|^{2}+d\right] \\
=\left\{\begin{array}{c}
\operatorname{Pr}\left[\left|h_{r d}\right|^{2} \geq \frac{c\left|h_{s r}\right|^{2}+d}{a\left|h_{s r}\right|^{2}-b\left|h_{s r}\right|^{2}}\right]=1,\left|h_{s r}\right|^{2}<\frac{b}{a} \\
\operatorname{Pr}\left[\left|h_{r d}\right|^{2} \leq \frac{c\left|h_{s r}\right|^{2}+d}{a\left|h_{s r}\right|^{4}-b\left|h_{s r}\right|^{2}}\right],\left|h_{s r}\right|^{2}>\frac{b}{a},
\end{array}\right.
\end{gathered}
$$


where $a=\eta P_{s}^{2} \rho(1-\rho) ; b=\eta P_{s} d_{s r}^{k} \widetilde{N}_{s r}\left(2^{2 R}-1\right) \rho ; c=P_{s} d_{s r}^{k} d_{r d}^{k} N_{r d}\left(2^{2 R}-1\right)(1-\rho)$; $d=d_{s r}^{2 k} d_{r d}^{k} \widetilde{N}_{s r} N_{r d}\left(2^{2 R}-1\right)$. (43) can be further rewritten as [6]:

$$
F_{C_{A F, \mathrm{EH}, n o d i r}}(R)=1-\int_{\frac{b}{a}}^{\infty} \lambda_{s r} \exp \left\{-\left(\lambda_{r d} \frac{c g+d}{(a g-b) g}+\lambda_{s r} g\right)\right\} d g,
$$

with the high SNR, $P_{S} \gg \widetilde{N}_{s r}$ and $P_{S} \gg N_{r d},(44)$ can be approximated as [6]:

$$
F_{C_{A F, \mathrm{EH}, n o \text { dir }}}(R) \approx 1-\exp \left\{-\frac{b \lambda_{s r}}{a}\right\} m \mathrm{~K}_{1}(m)=1-\exp \left\{-\frac{2^{2 R}-1}{\gamma_{s r, \mathrm{EH}, \text { mean }}}\right\} m \mathrm{~K}_{1}(m)
$$

where $m=\sqrt{\frac{4\left(2^{2 R}-1\right)}{\gamma_{r d} \mathrm{EH}, \text { mean }}}, \gamma_{r d, \mathrm{EH}, \text { mean }}=\frac{P_{s} \eta \rho}{d_{s r}^{k} d_{r d}^{k} N_{r d} \lambda_{s r} \lambda_{r d}}$ is the expected value of $\gamma_{r d, \mathrm{EH}}$, $\gamma_{s r}$,EH,mean $=\frac{P_{s}(1-\rho)}{d_{s r}^{k} N_{s r} \lambda_{s r}}$ is the expected value of $\gamma_{s r}$,EH. With (45), the optimization problem (41) can be further rewritten as:

$$
\min _{\substack{0 \leq d_{s r} \leq d_{s d} \\ 0 \leq \rho \leq 1}} 1-\exp \left\{-\frac{d_{s r}^{k} \widetilde{N}_{s r}\left(2^{2 R}-1\right) \lambda_{s r}}{P_{s}(1-\rho)}\right\} m \mathrm{~K}_{1}(m)
$$

Since the outage probability expression is too complicated to calculate the optimal relay position $\hat{d}_{s r}$ and optimal power splitting factor $\hat{\rho}$. Therefore, the optimal parameters $\hat{d}_{s r}$ and $\hat{\rho}$ are found by numerical analysis.

In the case, which consider with direct path, the optimization problem can be presented mathematically as:

$$
\min _{\substack{0 \leq d_{\text {sr }} \leq d_{\text {sd }} \\ 0 \leq \rho \leq 1}} P_{\text {out }}=\min _{\substack{0 \leq d_{s r} \leq d_{\text {sd }} \\ 0 \leq \rho \leq 1}} \operatorname{Pr}\left[C_{A F, \mathrm{EH}, d i r} \leq R\right],
$$

Subject to

$$
d_{s r}+d_{r d}=d_{s d}
$$

First, the $\mathrm{CDF}$ of $C_{A F, \mathrm{EH}, \text { no dir }}$ can be written as:

$$
\begin{gathered}
F_{C_{A F, \mathrm{EH}, \text { dir }}}(R)=\operatorname{Pr}\left[C_{A F, \mathrm{EH}, \text { dir }} \leq R\right]=\operatorname{Pr}\left[\gamma_{s d}+\gamma_{A F, \mathrm{EH}, \text { no dir }} \leq 2^{2 R}-1\right] \\
=\int_{0}^{\infty} \operatorname{Pr}\left[g+\gamma_{A F, \mathrm{EH}, \text { no dir }} \leq 2^{2 R}-1 \mid \gamma_{s d}=g\right] f_{\gamma_{s d}}(g) d g \\
=\int_{0}^{2^{2 R}}-1 \frac{1}{\gamma_{s d, \text { mean }}} \exp \left\{-\frac{g}{\gamma_{s d, \text { mean }}}\right\} \operatorname{Pr}\left[\gamma_{A F, \mathrm{EH}, \text { no dir }} \leq 2^{2 R}-1-g\right] d g \\
\approx \int_{0}^{2^{2 R}-1} \frac{1}{\gamma_{s d, \text { mean }}} \exp \left\{-\frac{g}{\gamma_{s d, \text { mean }}}\right\}\left(1-\exp \left\{-\frac{2^{2 R}-1-g}{\gamma_{s r, \mathrm{EH}, \text { meen }}}\right\} \bar{m} \mathrm{~K}_{1}(\bar{m})\right) d g \\
=1-\exp \left\{-\frac{2^{2 R}-1}{\gamma_{s d, \text { mean }}}\right\}-\int_{0}^{2^{2 R}} \frac{1}{\gamma_{s d, \text { mean }}} \exp \left\{-\frac{g}{\gamma_{s d, \text { mean }}}-\frac{2^{2 R}-1-g}{\gamma_{s r, \mathrm{EH}, \text { mean }}}\right\} \bar{m} \mathrm{~K}_{1}(\bar{m}) d g,
\end{gathered}
$$

where $\bar{m}=\sqrt{\frac{4\left(2^{2 R}-1-g\right)}{\gamma_{r d, \mathrm{EH}, \text { mean }}}}$. With (49), the optimization problem (47) can be further rewritten as:

$$
\begin{aligned}
\min _{0} 1-\exp \left\{-\frac{2^{2 R}-1}{\gamma_{s d, \text { mean }}}\right\}- \\
0 \leq d_{s r} \leq d_{s d} \\
0 \leq \rho \leq 1 \\
\int_{0}^{2^{2 R}-1} \frac{1}{\gamma_{s d, \text { mean }}} \exp \left\{-\frac{g}{\gamma_{s d, \text { mean }}}-\frac{2^{2 x}-1-g}{\gamma_{s r} \text { EH,mean }}\right\} \bar{m}_{R} \mathrm{~K}_{1}\left(\bar{m}_{R}\right) d g,
\end{aligned}
$$

Here also too difficult to calculate the optimal parameters $\hat{d}_{s r}$ and $\hat{\rho}$. Therefore, the optimal parameters $\hat{d}_{s r}$ and $\hat{\rho}$ are found by numerical analysis. 


\section{Numerical Results}

This section numerically analyzes the outage probabilities performance of cooperative communication with/without SWIPT under the strategy of amplify-and-forward. The commonly used parameters are set as Table 1. In addition, the signal to noise ratio (SNR) is defined as $P_{t} / N_{s r}$.

Table 1. Used parameters list.

\begin{tabular}{ccc}
\hline Data rate & $R$ & $1 \mathrm{bits} / \mathrm{s} / \mathrm{Hz}$ \\
Energy conversion efficiency & $\eta$ & 0.5 \\
Total transmit power & $P_{t}$ & 2 Joules $/ \mathrm{s}$ \\
Path loss exponent & $k$ & 2.7 \\
The distances from S to D & $d_{s d}$ & 2 \\
The variance of AWGN from S to RF band of EHR & $N_{s r, R}$ & 0.01 \\
The variance of AWGN from RF band to baseband & $N_{s r, C}$ & 0.01 \\
signal conversion at EHR & $N_{s r}=N_{s d}=N_{r d}$ & 0.02 \\
The variance of each path AWGN & $\sigma_{s r}^{2}=\sigma_{s d}^{2}=\sigma_{r d}^{2}$ & 1 \\
The variance of each path channel gains &
\end{tabular}

Figure 3 depicts the relationship between optimal outage probability and SNR with (26) and (27). It can be observed from Figure 3 that approximate expression (27) is acceptable when the SNR is more than $20 \mathrm{~dB}$. Therefore, (31), (29), and (30), which are derivate from approximate expression (27), can find the approximate optimal outage probability, the optimal relay position $\hat{d}_{s r}$ and optimal power allocation $\hat{P}_{s}$ with greatly reduce the computing complexity.

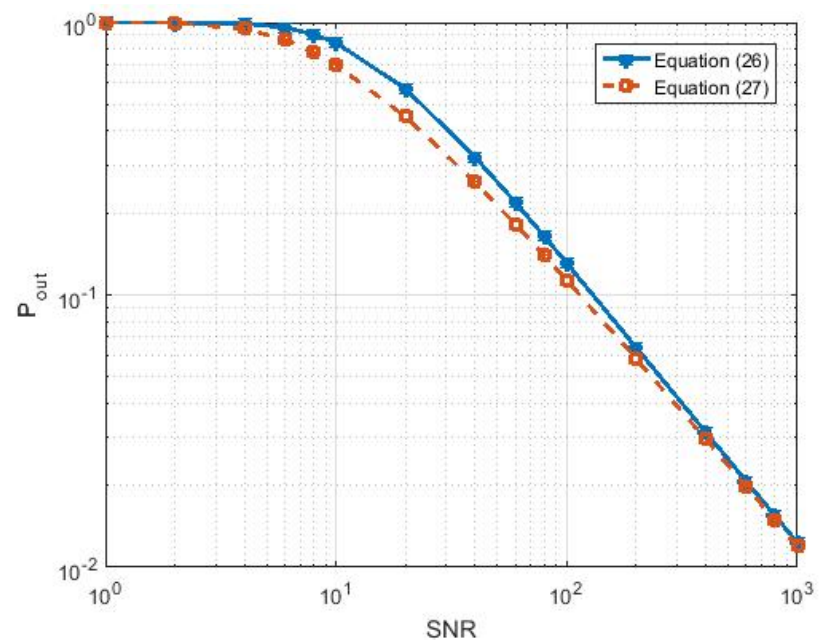

Figure 3. The optimal outage probability comparison of (26) and (27) with different SNR.

Figure 4 plots the optimal outage probability for (35) and (36) for different SNR, It can be seen from Figure 4 that the behavior of those two curves are similar when the SNR is more than $20 \mathrm{~dB}$. Furthermore, Figure 4 also shows that (36) cannot be approximated to (35) when the SNR is less than $11 \mathrm{~dB}$.

In Figure 5, we show the outage probability versus power splitting factor $\rho$ according to (45) with different $d_{s r}, 0.5,1$ and 1.5. It can be seen from (12) to (16) that the harvested energy increases as power splitting factor $\rho$ increases. However, the SNR of $y_{s r}$ EH will decrease when power splitting factor $\rho$ increases. The shorter $d_{s r}$ not only can offset the negative impact of using a larger power splitting factor $\rho$, but also can increase the harvested energy. Therefore, It can be seen from Figure 5 that the lowest outage probability occurs when power splitting factor $\rho$ approaches 1 with $d_{s r}=0.5$. With the increase of $d_{s r}$, the above two advantages are no longer available. If a large power splitting factor $\rho$ is selected when $d_{s r}$ is longer, the SNR of $y_{s r}, \mathrm{EH}$ will be worse, and there is not enough 
energy for acquisition. As a result, it also can be seen from Figure 5 that the optimal power splitting factor $\rho$ decreases as $d_{s r}$ increases.

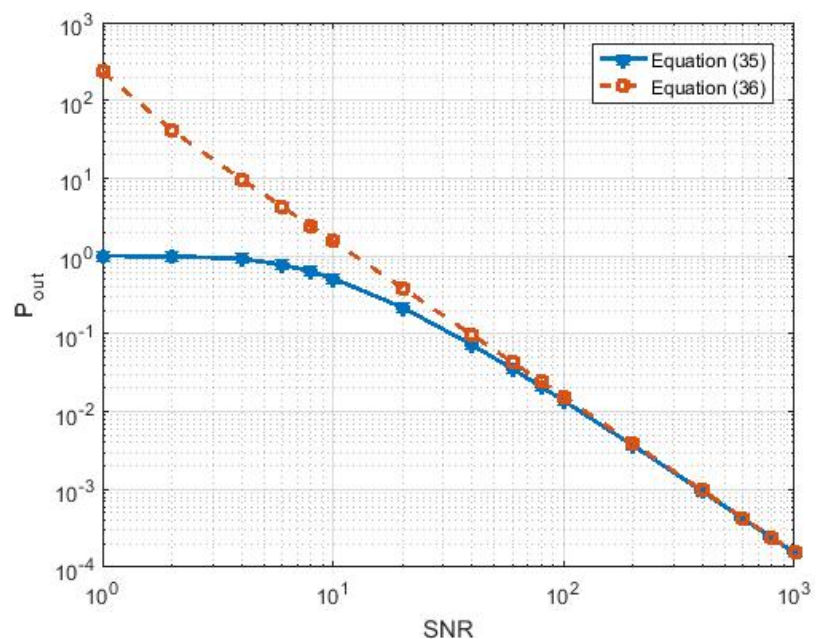

Figure 4. The optimal outage probability comparison of (35) and (36) with different SNR.

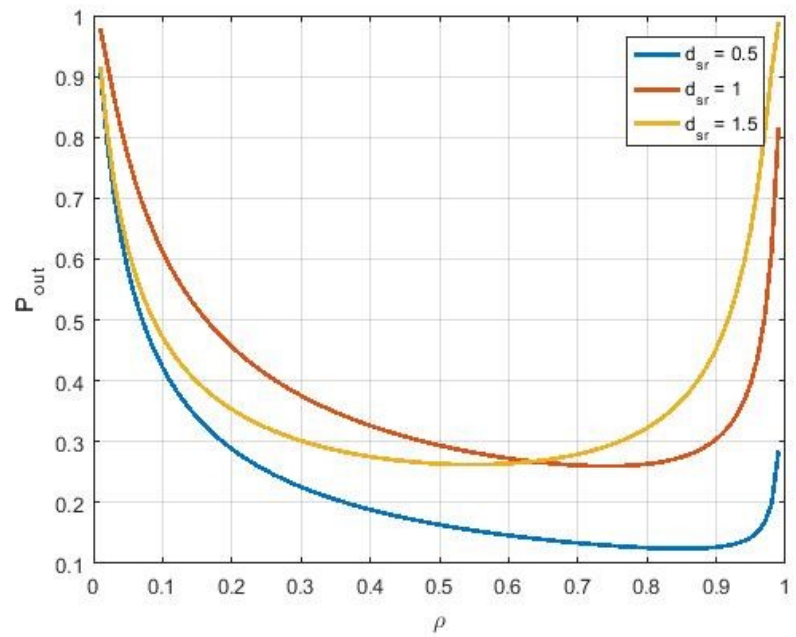

Figure 5. The outage probability with respect to the power splitting factor $\rho$ according to (45).

Figure 6 shows the outage probability of following four scenarios, conventional cooperative relay network without direct path, conventional cooperative relay network with direct path, cooperative relay network without direct path under SWIPT, and cooperative relay network with direct path under SWIPT, for different $d_{s r}$ under optimal power allocation $\hat{P}_{S}$ (or optimal power splitting factor $\hat{\rho}$ ). It can be seen from Figure 5 that the outage probability of cooperative relay network with SWIPT is greater than the outage probability of conventional cooperative relay network when $d_{s r}$ less than 0.5 without the direct path. In the case of considering the direct path, the outage probability of cooperative relay network with SWIPT is greater than the outage probability of conventional cooperative relay network when $d_{s r}$ less than 0.75 . Although the cooperative relay network with SWIPT compare to conventional cooperative relay network have higher outage probability in longer $d_{s r}$, however, considering the power source of the relay node, the use of energy harvesting has the advantages of no need to replace the battery or pull the power line, and it is a good application solution in the booming of the Internet of Things. 


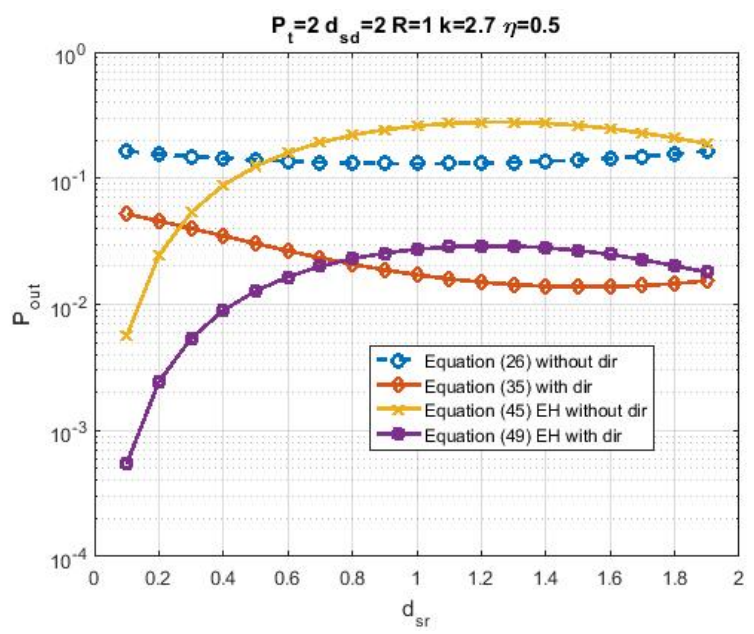

Figure 6. The outage probability with respect to S-R (or S-EHR) distance $d_{s r}$ under optimal $\rho$.

In Figure 7, we show the optimal outage probability versus SNR with six scenarios, conventional cooperative relay network without direct path, conventional cooperative relay network with direct path, cooperative relay network without direct path under SWIPT at $d_{s r}=0.5$, cooperative relay network without direct path under SWIPT at $d_{s r}=1$, cooperative relay network with direct path under SWIPT at $d_{s r}=0.5$ and cooperative relay network with direct path under SWIPT at $d_{s r}=1$. We consider the above scenarios of $d_{s r}=0.5$ for the case where the harvested energy is sufficient, and the scenarios of $d_{s r}=1$ are the case that the harvested energy is insufficient. The optimal outage probability of conventional cooperative relay network in Figure 7 comes from optimal relay position $\hat{d}_{s r}$ and optimal power allocation $\hat{P}_{S}$ under each different SNR and the optimal outage probability of cooperative relay network with SWIPT in Figure 7 comes from optimal power splitting factor $\hat{\rho}$ under each different SNR. It can be seen from Figure 7 that cooperative relay network without direct path under SWIPT at $d_{s r}=1$ has highest outage probability in the six scenarios for each SNR due to the distance of S-EHR is too long to harvested energy and there is no direct path assistance. Cooperative relay network without direct path under SWIPT at $d_{s r}=0.5$ and conventional cooperative relay network without direct path have similar optimal outage probability at SNR of $20 \mathrm{~dB}$, but as SNR increases, the optimal outage probability of Cooperative relay network without direct path under SWIPT at $d_{s r}=0.5$ is slightly higher than the other. Under consideration of joining the direct path, cooperative relay network with direct path under SWIPT at $d_{s r}=0.5$ which with sufficient harvested energy and conventional cooperative relay network with direct path have similar optimal outage probability.

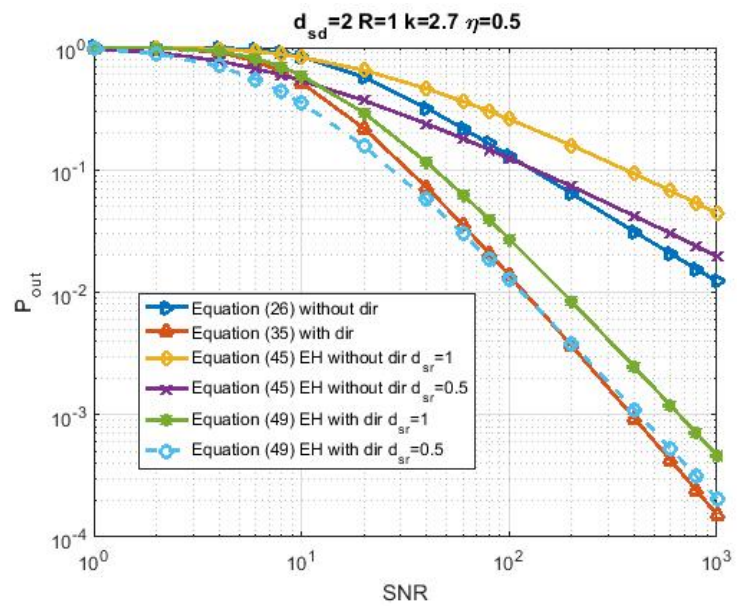

Figure 7. The optimal outage probability comparison under each different SNR. 


\section{Conclusions}

In the framework of cooperative communication, proper resource allocation can maximize the performance of the system. Based on this concept and the emerging issues of SWIPT, the research opportunities of this paper are carried out. The outage probabilities of cooperative relay networks with/without SWIPT under the strategy of amplify-andforward are carried out, and numerical results are compared. Numerical results show that cooperative relay networks with SWIPT are superior to cooperative relay networks without SWIPT under harvested sufficient energy. In addition, considering the special advantages of wireless charging with SWIPT, the system model of cooperative relay networks with SWIPT has market demand in the future.

Author Contributions: Z.-S.W., L.-H.L., J.-H.W., Y.-J.L. and C.-E.W. contributed equally to the literature review of the performance of both conventional cooperative relaying networks without SWIPT and cooperative relaying networks with SWIPT under an amplify-and-forward (AF) relaying network, then developed the first discussions about the different parameters of the proposed methodology and conducted the simulations and analysis of results. C.-E.W. guided the investigation and supervised this work. Z.-S.W. gave suggestions and guidance for the research. All authors have read and agreed to the published version of the manuscript.

Funding: This research received no external funding.

Data Availability Statement: The data used to support the finding of this study are included within the article.

Conflicts of Interest: The authors declare no conflict of interest.

\section{References}

1. Nosratinia, A.; Hunter, T.E.; Hedayat, A. Cooperative communication in wireless networks. IEEE Commun. Mag. 2004, 42, 74-80. [CrossRef]

2. Mahama, S.; Asiedu, D.K.P.; Lee, K.J. Simultaneous wireless information and power transfer for cooperative relay networks with battery. IEEE Access 2017, 5, 13171-13178. [CrossRef]

3. Rana, K.S. Contemporary work in energy harvesting \&importance of radiation free future 5-G WCN. In Proceedings of the 2018 Conference on Signal Processing and Communication Engineering Systems (SPACES), Vijayawada, India, 4-5 January 2018; pp. 12-15.

4. Chandravanshi, S.; Sarma, S.S.; Akhtar, M.J. Design of triple band differential rectenna for RF energy harvesting. IEEE Trans. Antennas Propag. 2018, 66, 2716-2726. [CrossRef]

5. Nasir, A.A.; Zhou, X.; Durrani, S.; Kennedy, R.A. Throughput and ergodic capacity of wireless energy harvesting based DF relaying network. In Proceedings of the 2014 IEEE International Conference on Communications (ICC), Sydney, Australia, 10-14 June 2014; pp. 4066-4071.

6. Nasir, A.A.; Zhou, X.; Durrani, S.; Kennedy, R.A. Relaying protocols for wireless energy harvesting and information processing. IEEE Trans. Wirel. Commun. 2013, 12, 3622-3636. [CrossRef]

7. Lee, H.; Song, C.; Choi, S.H.; Lee, I. Outage probability analysis and power splitter designs for SWIPT relaying systems with direct link. IEEE Commun. Lett. 2017, 21, 648-651. [CrossRef]

8. Ikhlef, A.; Bocus, M.Z. Outage performance analysis of relay selection in SWIPT systems. In Proceedings of the 2016 IEEE Wireless Communications and Networking Conference, Doha, Qatar, 3-6 April 2016; pp. 1-5.

9. Hossain, M.A.; Md Noor, R.; Yau, K.A.; Ahmedy, I.; Anjum, S.S. A Survey on Simultaneous Wireless Information and Power Transfer with Cooperative Relay and Future Challenges. IEEE Access 2019, 7, 19166-19198. [CrossRef]

10. Gautam, S.; Vu, T.X.; Chatzinotas, S.; Ottersten, B. Cache-Aided Simultaneous Wireless Information and Power Transfer (SWIPT) with Relay Selection. IEEE J. Sel. Areas Commun. 2018, 37, 187-201. [CrossRef]

11. Ojo, F.K.; Salleh, M.F.M. Energy efficiency optimization for SWIPT enabled cooperative relay networks in the presence of interfering transmitter. IEEE Commun. Lett. 2019, 23, 1806-1810. [CrossRef]

12. Zhou, X.; Zhang, R.; Ho, C.K. Wireless Information and Power Transfer: Architecture Design and Rate-Energy Tradeoff. IEEE Trans. Commun. 2013, 61, 4754-4767. [CrossRef]

13. Abramowitz, M.M.; Stegun, I.A. Handbook of Mathematical Functions with Formulas, Graphs, and Mathematical Tables; Dover: New York, NY, USA, 1965. 